\title{
MODELAGEM MATEMÁTICA E SIMULAÇÃO NUMÉRICA DO RESFRIAMENTO RÁPIDO DE MORANGO COM AR FORÇADO ${ }^{1}$
}

\author{
DANIELA C. Z. PIROZZI², MARIÂNGELA AMENDOLA ${ }^{3}$
}

RESUMO: Este trabalho abordou o resfriamento rápido com ar forçado de morango via simulação numérica. Para tanto, foi empregado o modelo matemático que descreve o processo de transferência de calor, com base na lei de Fourier, escrito em coordenadas esféricas e simplificado para descrever o processo unidimensional. A resolução da equação expressa pelo modelo matemático deu-se por meio da implementação de um algoritmo, fundamentado no esquema explícito do método numérico das diferenças finitas, executado no ambiente de computação científica MATLAB 6.1. A validação do modelo matemático foi realizada a partir da comparação de dados teóricos com dados obtidos num experimento, no qual morangos foram resfriados com ar forçado. Os resultados mostraram que esse tipo de investigação para a determinação do coeficiente de transferência de calor por convecção é promissora como ferramenta no suporte à decisão do uso ou desenvolvimento de equipamentos na área de resfriamento rápido de frutos esféricos com ar forçado.

PALAVRAS-CHAVE: métodos numéricos, transferência de calor, computação científica.

\section{MATHEMATICAL MODEL AND NUMERICAL SIMULATION OF STRAWBERRY FAST COOLING WITH FORCED AIR}

\begin{abstract}
This work approaches the forced air cooling of strawberry by numerical simulation. The mathematical model that was used describes the process of heat transfer, based on the Fourier's law, in spherical coordinates and simplified to describe the one-dimensional process. For the resolution of the equation expressed for the mathematical model, an algorithm was developed based on the explicit scheme of the numerical method of the finite differences and implemented in the scientific computation program MATLAB 6.1. The validation of the mathematical model was made by the comparison between theoretical and experimental data, where strawberries had been cooled with forced air. The results showed to be possible the determination of the convective heat transfer coefficient by fitting the numerical and experimental data. The methodology of the numerical simulations was showed like a promising tool in the support of the decision to use or to develop equipment in the area of cooling process with forced air of spherical fruits.
\end{abstract}

KEYWORDS: numerical methods, heat transfer, scientific computation.

\section{INTRODUÇÃO}

O uso de refrigeração no armazenamento de produtos agrícolas é recomendado para garantir o tempo de prateleira dos mesmos, pois reduz o metabolismo desses produtos, diminui a perda de água e controla o crescimento de microorganismos, os quais são prejudicados. Para que esse armazenamento refrigerado possa ser eficiente, deve-se levar em consideração, entre outros fatores, o resfriamento rápido imediatamente após a colheita (CHITARRA, 1999).

\footnotetext{
${ }^{1}$ Extraído da dissertação de mestrado do primeiro autor.

${ }^{2}$ Eng $^{\text {o }}$ Agrônomo, Doutorando, Departamento de Botânica, Instituto de Biociência, UNESP, Botucatu - SP, Fone: (0XX14)3811.6265, dcpirozzi@yahoo.com

${ }^{3}$ Matemática, Profa. Doutora, FEAGRI-UNICAMP, Campinas - SP.
}

Recebido pelo Conselho Editorial em: 21-10-2003

Aprovado pelo Conselho Editorial em: 24-2-2005 
A proposição e o uso da tecnologia de resfriamento rápido com ar forçado de produtos perecíveis necessita de aprofundamento no conhecimento dos processos físicos que ocorrem durante esse resfriamento, o que pode ser realizado tanto por meio de investigação experimental, quanto por estudos teóricos por meio do emprego de métodos matemáticos, sendo esses últimos, somente recentemente, utilizados na FEAGRI/UNICAMP.

Segundo INCROPERA \& DE WITT (1996), os métodos matemáticos empregados para o estudo de resfriamento expressam, em geral, problemas de condução transiente, com muitas geometrias e condições de contorno simplificadas e podem ser resolvidos por meio de métodos analíticos ou numéricos.

Quando é possível obter as soluções analíticas, essas aparecem na forma de uma série infinita, que para serem calculadas devem ser truncadas com um número determinado de termos. Uma vez que essas séries infinitas são truncadas, elas passam a fornecer soluções aproximadas, assim como aquelas obtidas pelo uso de métodos numéricos.

ALVAREZ \& TRYSTRAM (1995) simularam numericamente o resfriamento rápido com ar forçado de frutos esféricos, utilizando modelo matemático baseado na lei de Fourier, e mostraram que modelos matemáticos e simulação numérica são ótimas ferramentas, tanto para o projeto, quanto para o uso do resfriamento.

Para a validação dos resultados gerados, via simulação numérica do processo de resfriamento rápido com ar forçado, são necessários as medições experimentais de temperatura e o conhecimento de parâmetros físicos do mesmo. Dentre os parâmetros físicos, um é limitante, o coeficiente de transferência de calor por convecção, denotado por $h$, é sensível aos parâmetros do processo experimental, como velocidade e propriedades térmicas do ar de resfriamento, geometria do sistema, local onde a temperatura do fluido é medida, entre outros.

Nesse sentido, CASTRO \& AMENDOLA (1999), TERUEL (2000) e AMENDOLA \& TERUEL (2002), usando modelos matemáticos e métodos numéricos distintos, simularam o resfriamento rápido com ar forçado de laranjas e estimaram o valor de $h$, ajustando os dados encontrados numericamente aos dados obtidos via experimento prático. Essa metodologia também pode ser vista em processos de secagem, como em QUEIROZ \& NEBRA (2002) e ITO \& AMENDOLA (2002), que a usaram para estimar o coeficiente de difusão em secagem de bananas e soja, respectivamente.

Os resultados encontrados nos trabalhos citados anteriormente justificam o cumprimento do objetivo do presente trabalho, que foi selecionar um modelo matemático e desenvolver método numérico para simular o resfriamento rápido com ar forçado de morango para obter o respectivo coeficiente de transferência de calor por convecção.

\section{MATERIAL E MÉTODOS}

Os dados experimentais utilizados neste trabalho foram extraídos do experimento realizado por BINOTTI (2000), no qual morangos sofreram o resfriamento rápido com ar forçado. Além desses dados experimentais, foram extraídos da literatura dados de propriedades termofísicas do morango, como condutividade térmica $\left(\mathrm{k}_{\mathrm{p}}\right)$ igual a $0,54 \mathrm{~W} \mathrm{~m}^{-1}{ }^{\circ} \mathrm{C}^{-1}$ e difusividade térmica $(\alpha)$ igual a $1,7210^{-7} \mathrm{~m}^{2} \mathrm{~s}^{-1}$, extraídos de MILES et al. (1983), citados por JANCSÓK et al. (2002).

No experimento realizado por BINOTTI (2000), foram utilizados: a câmara fria do laboratório de termodinâmica da Faculdade de Engenharia Agrícola - FEAGRI, da Universidade Estadual de Campinas UNICAMP, um computador, o programa de aquisição de dados - AQDADOS, termopares do tipo T, caixas de papelão e morangos da variedade "Campinas IAC-2712". A partir desse experimento, pode-se extrair: a temperatura inicial $\left(\mathrm{T}_{\mathrm{i}}\right)$ no centro de 12 morangos, nos quais a menor temperatura inicial medida foi igual a $13,8{ }^{\circ} \mathrm{C}$ e a maior igual a $17,2{ }^{\circ} \mathrm{C}$; a temperatura do ar de resfriamento $\left(\mathrm{T}_{\mathrm{a}}\right)$ foi de $0{ }^{\circ} \mathrm{C}$; o raio médio $(\mathrm{R})$ dos morangos foi de $0,025 \mathrm{~m}$, obtido a partir da média do maior diâmetro longitudinal dos 
morangos, e os dados da temperatura no centro do morango $\left(\mathrm{T}_{\exp }\right)$ medidos ao longo do resfriamento, os quais foram utilizados para a validação dos dados numéricos e ajuste do valor do coeficiente de transferência de calor por convecção (h).

Para obter os dados de temperatura no interior dos morangos ao longo do resfriamento, foram tomados 12 frutos aleatoriamente, nos quais foram introduzidos os termopares o mais próximo possível do centro. Desses dados de temperatura, puderam-se obter 12 "curvas" experimentais (denomina-se "curvas" a dispersão desses dados). A partir dessas "curvas", foram tomadas aleatoriamente duas, uma para a escolha do melhor tipo de ajuste das "curvas" experimentais e outra para a avaliação da influência da malha. Para a validação da simulação numérica e ajuste do valor do coeficiente de transferência de calor por convecção (h), foram tomadas, também aleatoriamente, seis das "curvas" experimentais.

Para determinar o melhor ajuste para os dados experimentais, foram aplicados os ajustes exponencial e polinomiais de $2^{\circ} ; 3^{-} ; 4^{\circ}$ e $6^{-}$graus na "curva" experimental selecionada. A escolha da equação que seria a empregada para ajustar os dados experimentais foi feita com base tanto na representatividade do fenômeno físico em questão, quanto no valor do resíduo gerado quando comparada com uma das "curvas" obtidas experimentalmente.

Segundo BOLDRINI et al. (1980), para o cálculo desse resíduo, pode-se aplicar a norma da diferença entre os dados reais e os apresentados, que é dada pela seguinte expressão:

$$
\left\|\mathrm{T}_{\exp }-\mathrm{T}_{\text {num }}\right\|=\sqrt{\sum_{\mathrm{i}}^{\mathrm{n}}\left(\mathrm{T}_{\exp }(\mathrm{t})-\mathrm{T}_{\text {num }}(\mathrm{t})\right)^{2}}
$$

em que,

$\mathrm{T}_{\text {exp }}$ - temperatura no centro do morango, obtida experimentalmente, ${ }^{\circ} \mathrm{C}$;

$\mathrm{T}_{\text {num }}$ - temperatura no centro do morango, obtida segundo a equação do ajuste, ${ }^{\circ} \mathrm{C}$;

$\mathrm{n}$ - total de medidas, e

$\mathrm{t}$ - tempo, s.

O modelo matemático associado ao processo de resfriamento de frutos esféricos e selecionado para o desenvolvimento dessa pesquisa é o que aparece em TRELEA et al. (1998), com base na lei de Fourier e que descreve o processo, transferência de calor em frutos esféricos, que, escrito para determinação de $\mathrm{T}(\mathrm{r}, \mathrm{t})$, é expresso por :

$$
\frac{\partial \mathrm{T}}{\partial \mathrm{t}}(\mathrm{r}, \mathrm{t})=\alpha\left(\frac{2}{\mathrm{r}} \frac{\partial \mathrm{T}}{\partial \mathrm{r}}(\mathrm{r}, \mathrm{t})+\frac{\partial^{2} \mathrm{~T}}{\partial \mathrm{r}^{2}}(\mathrm{r}, \mathrm{t})\right) ; \quad \mathrm{t} \geq 0, \quad \mathrm{r} \in[0, \mathrm{R}]
$$

em que,

$\mathrm{T}(\mathrm{r}, \mathrm{t})$ - temperatura no interior do morango ao longo do tempo, ${ }^{\circ} \mathrm{C}$;

$\mathrm{t}$ - tempo, s;

$\mathrm{r}$ - distância $\mathrm{r}$ do centro do morango, m;

$\alpha$ - difusividade térmica do morango, $\mathrm{m}^{2} \mathrm{~s}^{-1}$;

$\mathrm{k}_{\mathrm{p}}$ - condutividade térmica do morango, $\mathrm{W} \mathrm{m}^{-1}{ }^{\circ} \mathrm{C}^{-1}$;

$\mathrm{C}_{\mathrm{p}}$ - calor específico do morango, $\mathrm{J} \mathrm{kg}^{-1}{ }^{\circ} \mathrm{C}^{-1}$;

$\rho$ - densidade do morango, $\mathrm{kg} \mathrm{m}^{-3}$, e

$\mathrm{R}$ - raio do morango, $\mathrm{m}$.

A condição inicial associada é:

$$
\mathrm{T}(\mathrm{r}, 0)=\mathrm{T}_{0} ; \quad \mathrm{r} \in[0, \mathrm{R}]
$$

As condições de contorno são: 


$$
\begin{aligned}
& \frac{\partial \mathrm{T}}{\partial \mathrm{r}}(0, \mathrm{t})=0 ; \quad \mathrm{t} \geq 0 \\
& -\mathrm{k}_{\mathrm{p}} \frac{\partial \mathrm{T}}{\partial \mathrm{r}}(\mathrm{R}, \mathrm{t})=\mathrm{h}\left[\mathrm{T}_{\mathrm{s}}(\mathrm{t})-\mathrm{T}_{\mathrm{a}}(\mathrm{t})\right] ; \mathrm{t} \geq 0
\end{aligned}
$$

em que,

$\mathrm{h}$ - coeficiente de transferência de calor por convecção, $\mathrm{W} \mathrm{m}{ }^{-2}{ }^{\circ} \mathrm{C}^{-1}$;

$\mathrm{T}_{\mathrm{s}}$ - temperatura na superfície do morango, ${ }^{\circ} \mathrm{C}$;

$\mathrm{T}_{\mathrm{a}}$ - temperatura na câmara de resfriamento, ${ }^{\circ} \mathrm{C}$;

$\mathrm{k}_{\mathrm{p}}$ - condutividade térmica do morango, $\mathrm{W} \mathrm{m}^{-1}{ }^{\circ} \mathrm{C}^{-1}, \mathrm{e}$

$\mathrm{R}$ - raio do morango, $\mathrm{m}$.

O método numérico selecionado para a resolução da equação presente no modelo matemático foi o método explícito das diferenças finitas (RICHTMYER \& MORTON, 1967). A escolha desse método numérico foi baseada no trabalho de PIROZZI \& AMENDOLA (2002), já que os resultados mostraram uma diferença inferior a $10 \%$ entre os dados de origem numérica e experimental. Utilizando esse método, que é um método condicionalmente instável, é possível escrever a forma discretizada da eq.(2).

O primeiro passo para discretizar a eq.(2), sujeita às condições (3), (4) e (5), é estabelecer uma convenção que, no caso, será:

$$
\mathrm{T}_{\mathrm{i}}^{\mathrm{n}} \equiv \mathrm{T}(\mathrm{i} \Delta \mathrm{r}, \mathrm{n} \Delta \mathrm{t}) \quad ; \quad \text { para } \mathrm{i}=[1, \mathrm{nx}] \text { e } \mathrm{n}=[1, \mathrm{nt}]
$$

em que,

i - localização dos pontos de resolução na direção radial;

nx - último ponto de resolução na direção radial;

$\Delta \mathrm{r}$ - distância entre os pontos de resolução na direção radial, m;

$\mathrm{n}$ - número total de passos no tempo;

$\Delta \mathrm{t}$ - incremento de tempo, min, e

nt - último passo no tempo.

A semidiscretização no tempo da eq.(2), por uma fórmula de diferenças finitas avançadas, com erro da ordem de $(\Delta t)$, é:

$$
\left(\frac{\partial \mathrm{T}}{\partial \mathrm{t}}(\mathrm{r}, \mathrm{t})\right)_{i}^{n} \cong\left(\frac{\mathrm{T}_{\mathrm{i}}^{\mathrm{n}+1}-\mathrm{T}_{\mathrm{i}}^{\mathrm{n}}}{\Delta \mathrm{t}}\right)
$$

A semidiscretização no espaço do primeiro termo do lado direito da eq.(2), por uma fórmula de diferenças finitas avançadas, para o caso explícito e com erro da ordem de $(\Delta \mathrm{r})$, é:

$$
\left(\frac{\partial \mathrm{T}}{\partial r}(\mathrm{r}, \mathrm{t})\right)_{i}^{n} \cong\left(\frac{\mathrm{T}_{\mathrm{i}+1}^{\mathrm{n}}-\mathrm{T}_{\mathrm{i}}^{\mathrm{n}}}{\Delta \mathrm{r}}\right)
$$

A semidiscretização no espaço do segundo termo do lado direito da eq.(4), por uma fórmula de diferenças finitas centradas, para o caso explícito e com erro da ordem de $\left(\Delta \mathrm{r}^{2}\right)$, é:

$$
\left(\frac{\partial^{2} \mathrm{~T}}{\partial r^{2}}(\mathrm{r}, \mathrm{t})\right)_{i}^{n} \cong\left(\frac{\mathrm{T}_{\mathrm{i}-1}^{\mathrm{n}}-\mathrm{T}_{\mathrm{i}}^{\mathrm{n}}+T_{i+1}^{n}}{\Delta \mathrm{r}^{2}}\right)
$$

Denotando-se 


$$
\mathrm{F}=\left(\alpha \frac{\Delta \mathrm{t}}{\Delta \mathrm{r}^{2}}\right)
$$

a forma discretizada da eq.(4) é:

$$
\mathrm{T}_{\mathrm{i}}^{\mathrm{n}+1}=\mathrm{F} \mathrm{T}_{\mathrm{i}-1}^{\mathrm{n}}+\left(1-2 \mathrm{~F}-\frac{2 \mathrm{~F}}{\mathrm{i}}\right) \mathrm{T}_{\mathrm{i}}^{\mathrm{n}}+\left(\mathrm{F}+\frac{2 \mathrm{~F}}{\mathrm{i}}\right) \mathrm{T}_{\mathrm{i}+1}^{\mathrm{n}}
$$

A discretização da condição inicial (3) é dada por:

$$
\mathrm{T}_{\mathrm{i}}^{\mathrm{n}}=\mathrm{T}_{1} \quad ; \quad \mathrm{i}=1 \ldots \mathrm{nx} ; \quad \mathrm{n}=1 ;
$$

A discretização da condição de contorno (4) é dada por:

$$
\left(\frac{\partial T}{\partial r}(1, t)\right)_{i}^{n} \cong \frac{T_{i+1}^{n}-T_{i}^{n}}{\Delta r}=0 \quad ; \quad \forall \mathrm{n} \geq 1 ; \quad \mathrm{i}=1 ;
$$

ou

$\mathrm{T}_{2}^{\mathrm{n}}=\mathrm{T}_{1}^{\mathrm{n}} \quad ; \quad \forall \mathrm{n} \geq 1 \quad ; \quad \mathrm{i}=1 \quad ;$

Analogamente, a discretização da condição de contorno (5) é dada por:

$$
\begin{aligned}
& \frac{\mathrm{T}_{\mathrm{nx}}^{\mathrm{n}}-\mathrm{T}_{\mathrm{nx}-1}^{\mathrm{n}} \cong\left(\frac{-\mathrm{h}}{\mathrm{kp}}\right)\left[\mathrm{T}_{\mathrm{nx}}^{\mathrm{n}}-\mathrm{T}_{\mathrm{a}}\right]}{\Delta \mathrm{r}} \quad ; \quad \mathrm{n}=1 \ldots \mathrm{nt} ; \\
& \mathrm{T}_{\mathrm{nx}}^{\mathrm{n}}=\frac{\mathrm{T}_{\mathrm{nx}-1}^{\mathrm{n}}+\left(\frac{\mathrm{h}}{\mathrm{kp}}\right) \Delta \mathrm{rT}_{\mathrm{a}}}{1+\left(\frac{\mathrm{h}}{\mathrm{kp}}\right) \Delta \mathrm{r}} \quad ; \quad \mathrm{n}=1 \ldots \mathrm{nt} \quad ;
\end{aligned}
$$

Após selecionar o modelo matemático, o método numérico e garantir a estabilidade do método numérico, evitando assim possíveis erros ou perturbações que poderiam mascarar e até prejudicar os resultados, foi feita a elaboração de um algoritmo, sendo esse implementado no MATLAB 6.1. Sequiencialmente, foram realizadas as simulações numéricas, primeiro para a avaliação da influência da malha, e segundo para o ajuste do $h$.

Para avaliar a influência da malha nos resultados numéricos, tomou-se $\Delta \mathrm{r}=\mathrm{R} / 5$, em que $\Delta \mathrm{r}$ é a distância entre os pontos de resolução na direção radial, $m$, e cujos resultados podem ser obtidos por meio do uso de calculadora. Em seguida, $\Delta \mathrm{r}=\mathrm{R} /(5 \mathrm{~L})$, para $\mathrm{L}=2,3,4 \ldots 16$, cujos resultados são obtidos com o uso do computador. Em cada um desses casos, os valores da temperatura simulada ao longo do tempo no ponto discreto da malha espacial considerada mais próxima do centro do fruto: $\mathrm{i}=2$, quando $\Delta \mathrm{r}=\mathrm{R} / 5$, foram coletados e, posteriormente, colocados num gráfico. A seleção da malha a ser considerada nas simulações numéricas e o ajuste do valor de $h$ foram realizados segundo o cálculo do resíduo entre duas simulações subseqüientes. Para o cálculo do resíduo, foi utilizada a eq.(1).

Selecionada a melhor malha, pode-se dar início às simulações numéricas e ao ajuste do valor de $h$. Para determinar qual o valor de $h$ mais representativo para cada "curva" experimental, foram realizadas simulações numéricas, variando-se o valor de $h$ a partir do cálculo do resíduo entre os dados numéricos e os experimentais. O cálculo do resíduo foi feito utilizando a eq.(1).

Feitos a seleção da melhor "curva" numérica e o respectivo valor de $h$ para cada "curva" experimental, pode-se calcular o tempo de 1/2 e 7/8 do resfriamento teórico em cada simulação numérica. 
O cálculo da temperatura no tempo de 1/2 e 7/8 do resfriamento é realizado por meio da taxa adimensional de temperatura (TAT) (MITCHEL, 1992), que é dada pelas expressões:

$$
\begin{aligned}
& \operatorname{TAT}(1 / 2)=\frac{\operatorname{Tce}-\operatorname{Tar}}{\operatorname{Tin}-\operatorname{Tar}}=(1-1 / 2) \\
& \operatorname{TAT}(7 / 8)=\frac{\operatorname{Tce}-\operatorname{Tar}}{\operatorname{Tin}-\operatorname{Tar}}=(1-7 / 8)
\end{aligned}
$$

em que,

Tce - temperatura no centro do morango ao longo do resfriamento, ${ }^{\circ} \mathrm{C}$;

Tar - temperatura do ar de resfriamento, ${ }^{\circ} \mathrm{C}$, e

Tin - temperatura inicial no centro do morango, ${ }^{\circ} \mathrm{C}$.

\section{RESULTADOS E DISCUSSÃO}

Dentre os ajustes realizados para os dados experimentais, o de menor resíduo foi o polinomial de sexta ordem; no entanto, a "curva" que melhor representou o fenômeno físico do processo foi a do ajuste polinomial de quarta ordem, sendo esse o ajuste selecionado.

Pela Tabela 1, pode-se observar que, no refinamento da malha, o resíduo entre as curvas numéricas diminuiu. A partir de 31 até 71 pontos discretos na malha, os resíduos são todos da ordem de $10^{-1}$ e para aumentar a precisão para $10^{-2}$ só acima de 76 pontos discretos na malha. Entretanto, na Figura 1, é possível verificar que, a partir de 30 pontos discretos na malha, não há mais influência da mesma.

TABELA 1. Resíduo entre cada par de curva numérica, obtido com diferentes pontos discretos na malha (nx).

\begin{tabular}{cccccc}
\hline $\mathrm{nx}$ & Resíduo & $\mathrm{nx}$ & Resíduo & $\mathrm{nx}$ & Resíduo \\
\hline $6 \times 86$ & 1,0429 & $36 \times 86$ & 0,0739 & $66 \times 86$ & 0,0157 \\
$11 \times 86$ & 0,4214 & $41 \times 86$ & 0,0579 & $71 \times 86$ & 0,0109 \\
$16 \times 86$ & 0,2511 & $46 \times 86$ & 0,0457 & $76 \times 86$ & 0,0069 \\
$21 \times 86$ & 0,1716 & $51 \times 86$ & 0,0360 & $81 \times 86$ & 0,0032 \\
$26 \times 86$ & 0,1256 & $56 \times 86$ & 0,0280 & & \\
$31 \times 86$ & 0,0955 & $61 \times 86$ & 0,0213 & & \\
\hline
\end{tabular}

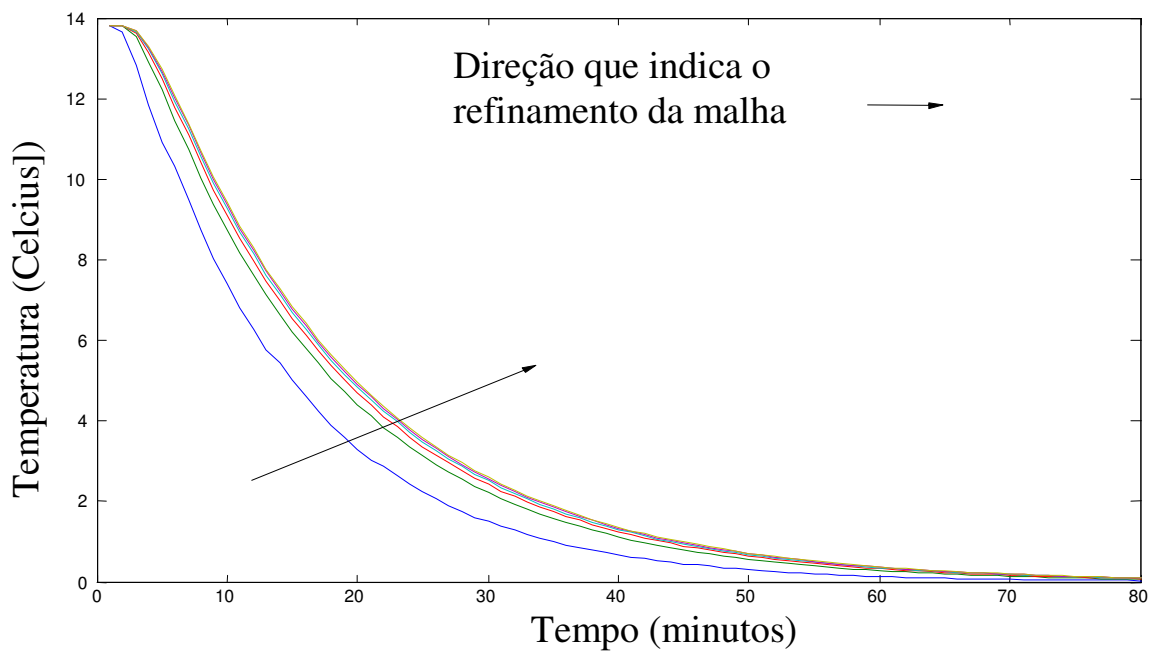

FIGURA 1. Curvas numéricas da variação da temperatura do morango, ao longo do tempo de resfriamento rápido, obtidas com $\mathrm{nx}=6$ a 86 , para o morango $\mathrm{n}^{\mathrm{o}} 7 \mathrm{e} \mathrm{h}=19,578 \mathrm{~W} \mathrm{~m}^{-2} \mathrm{C}^{-1}$. 
Quanto ao ajuste do valor de $h$, as simulações numéricas foram realizadas com valor de $h$ inicialmente igual a $10 \mathrm{~W} \mathrm{~m}^{-2}{ }^{\circ} \mathrm{C}^{-1}$, até que se obtivesse o menor resíduo entre curvas experimentais e numéricas. Na Tabela 2, são mostrados os valores dos menores resíduos obtidos entre as curvas experimentais e numéricas do morango e seus respectivos valores de $h$. Nessa tabela, pode-se verificar, também, que é possível obter diferentes valores de $h$ dentro de uma mesma caixa durante um processo de resfriamento rápido de ar forçado. Isso ocorre, pois o valor do coeficiente de transferência de calor por convecção não depende apenas de um fator, mas de um conjunto de fatores associados. Isso pode ser observado também, por exemplo, em TERUEL (2000) que, avaliando três pontos dentro de uma mesma caixa, encontrou valores diferentes de $h$ para cada ponto, em que a menor diferença entre eles foi de $29 \%$ e a maior de $62 \%$.

TABELA 2. Valores dos menores resíduos obtidos entre as "curvas" de temperatura experimental e numérica para cada morango, e seus respectivos valores de $h$.

\begin{tabular}{lcccccc}
\hline Morangos & 1 & 2 & 3 & 4 & 5 & 6 \\
\hline Valores dos menores resíduos & 1,1850 & 1,5971 & 1,0864 & 1,5484 & 1,1884 & 1,1805 \\
$h\left[\mathrm{~W} \mathrm{~m}^{-2} \mathrm{C}^{-1}\right]$ & 21 & 46 & 16 & 39 & 21 & 14 \\
\hline
\end{tabular}

Um exemplo de como foi realizado o resfriamento rápido com ar forçado de morangos e determinado o valor de $h$ para diferentes velocidades do ar de resfriamento é apresentado em FIKIIN et al. (1999). Para realizar o experimento, utilizaram tanto a temperatura do ar de resfriamento, quanto as caixas de acondicionamento dos morangos, próximas às de BINOTTI (2000) e reduziram a temperatura dos frutos num total de $22{ }^{\circ} \mathrm{C}$. Os autores apresentaram resultados nos quais os valores de $h$, obtidos para valores da velocidade do ar de resfriamento inferiores a $1 \mathrm{~m} \mathrm{~s}^{-1}$, foram inferiores a $20 \mathrm{~W} \mathrm{~m}^{-2}{ }^{\circ} \mathrm{C}^{-1}$, e para atingir valores de $h$ acima de $25 \mathrm{~W} \mathrm{~m}^{-2} \mathrm{C}^{-1}$, foi necessária uma velocidade do ar de resfriamento em torno de $2,5 \mathrm{~m} \mathrm{~s}^{-1}, \mathrm{o}$ que chega a ser quatro vezes superior às utilizadas por BINOTTI (2000), que foi de $0,8 \mathrm{~m} \mathrm{~s}^{-1}$.

$\mathrm{Na}$ Tabela 3, são apresentados os tempos numéricos e experimentais para 1/2 e 7/8 do resfriamento do morango 3 para $\mathrm{h}=16 \mathrm{~W} \mathrm{~m}^{-2} \mathrm{C}^{-1}$, em que se pode notar uma diferença de dois minutos para o tempo de $1 / 2$ do resfriamento e de 21 minutos para tempo de $7 / 8$ do resfriamento.

TABELA 3. Comparação do tempo de $1 / 2$ e 7/8 do resfriamento numérico e experimental - morango 3, com valor de $\mathrm{h}=16 \mathrm{~W} \mathrm{~m}^{-2} \mathrm{C}^{-1}$.

\begin{tabular}{lcc}
\hline & $1 / 2$ resfriamento (min) & $7 / 8$ do resfriamento (min) \\
\hline Dados numéricos & 25,8 & 65,9 \\
Dados experimentais & 23,8 & 87,2 \\
\hline
\end{tabular}

Na Figura 2, é apresentada a curva que mostrou o menor resíduo, quando comparadas as curvas de temperatura de origem numérica e experimental e o respectivo valor de $h$ ajustado.

Uma vez que, para o morango 3, se obteve o menor resíduo quando comparadas as "curvas" de origem numérica e experimental, pode-se, por exemplo, estabelecer que o morango e seu respectivo $h$ sejam representativos do processo e, com essa estimativa, pode-se simular o processo de transferência de calor no interior do fruto e calcular o tempo que o mesmo demora para atingir certa temperatura, o que, em termos práticos, significa maior controle do processo de resfriamento.

Por exemplo, na Figura 3, pode ser vista a simulação numérica da variação da temperatura ao longo do resfriamento em seis pontos do raio do morango 3. O comportamento previsto para a variação da temperatura ao longo do raio do morango, no resfriamento rápido com ar forçado, é que, quanto mais próximo da superfície do fruto, mais rápido se dá o processo. Nota-se que a coluna ao lado do gráfico relaciona cor com temperatura em graus Celsius. 


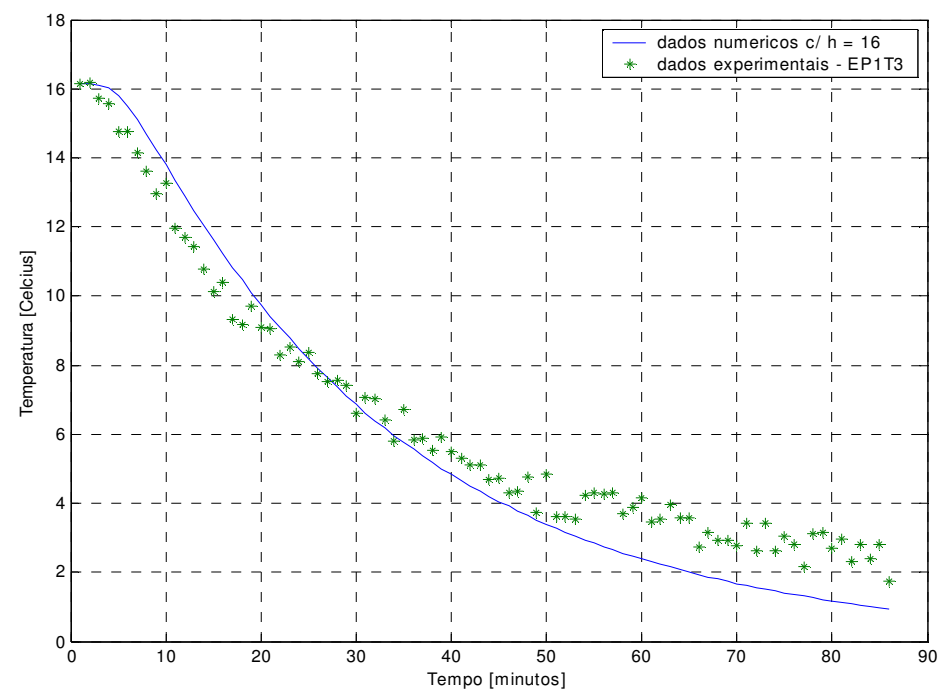

FIGURA 2. Variação da temperatura ao longo do tempo de resfriamento segundo dados experimentais e numéricos para o morango 3, com valor de $\mathrm{h}=16 \mathrm{~W} \mathrm{~m}^{-2} \mathrm{C}^{-1}$.

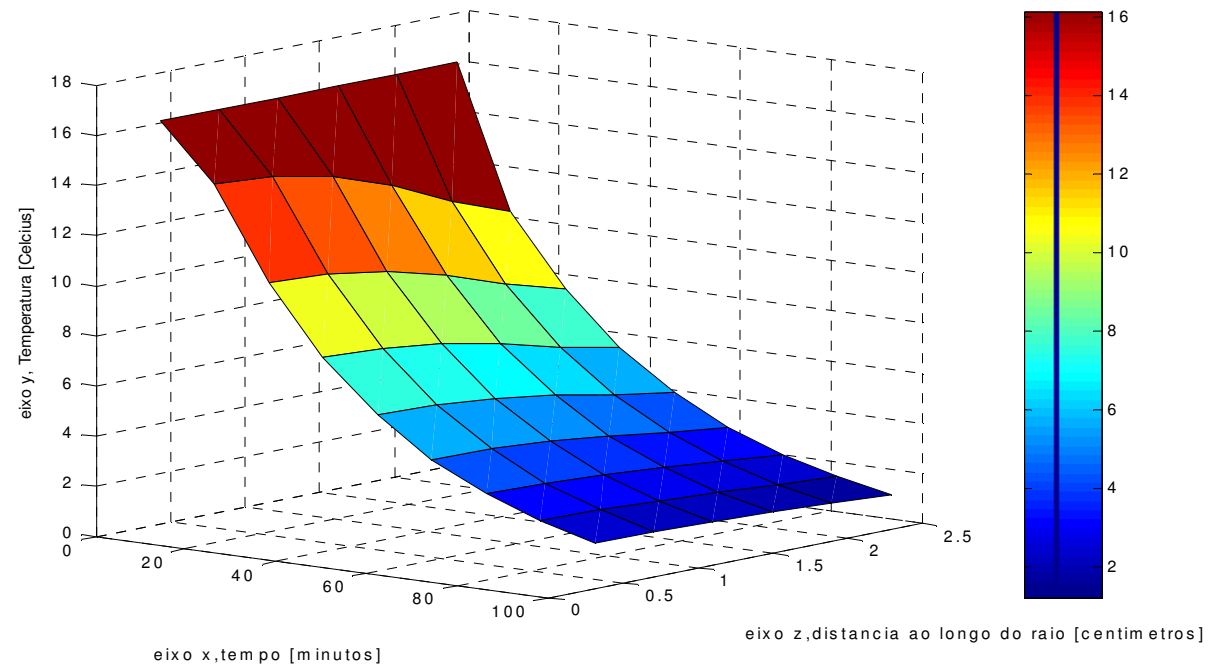

FIGURA 3. Variação da temperatura ao longo do resfriamento em seis pontos do raio do morango 3, com valor de $\mathrm{h}=16 \mathrm{~W} \mathrm{~m}^{-2} \mathrm{C}^{-1}$.

\section{CONCLUSÕES}

A metodologia das simulações numéricas mostrou-se promissora como ferramenta no suporte à decisão do uso ou desenvolvimento de equipamentos na área de resfriamento rápido com ar forçado de frutos esféricos, uma vez que foi capaz de fornecer subsídios para a estimação do valor do coeficiente convectivo de transferência de calor e determinação do tempo de resfriamento do morango. No caso do método numérico explícito usado, deve-se observar a condição de estabilidade.

\section{AGRADECIMENTOS}

Ao Prof. Luís Augusto Barbosa Cortez e Cibele Binotti, por cederem gentilmente os dados de natureza experimental. 


\section{REFERÊNCIAS}

ALVAREZ, G.;TRYSTAM, G. Designer of a new strategy for the control of the refrigeration process: fruit and vegetables conditioned in a pallet. Food Control, Guilford, v.6, n.6, p.347-55,1995.

AMENDOLA, M.;TERUEL, B. Uso de um esquema implícito y de splines para la simulación numérica Del processo de enfriamento de naranjas. Simiente, Santiago, v.72, n.3-4, p.141.

BINOTTI, C.S. Avaliação quantitativa do método de resfriamento rápido com ar forçado para morango. 2000. 83 f. Dissertação (Mestrado em Tecnologia Pós-Colheita) - Faculdade de Engenharia Agrícola, Universidade Estadual de Campinas, Campinas, 2000.

BOLDRINI, J.L.; RODRIGUES COSTA, S.I.; FIGUEIREDO, V.L.; WETZLER,H.G. Álgebra linear. 3.ed. São Paulo: Harper \& Row do Brasil, 1980. 411 p.

CASTRO, L.R.; AMENDOLA, M. Simulação numérica do processo de transferência de calor em vegetais esféricos. In: CONGRESSO IBERO-LATINO-AMERICANO EM MÉTODOS COMPUTACIONAIS E ENGENHARIA, 20., 1999, São Paulo. Anais... São Paulo. 1 CD ROM. CHITARRA, A.B. Armazenamento de frutos e hortaliças por refrigeração. Lavras: FAEPE, 1999. $58 \mathrm{p}$.

FIKIIN, A.G.; FIKIIN, K.A.;TRIPHONOV, S.D. Equivalent thermophysical properties and surface heat transfer coefficient of fruits layers in trays during cooling. Journal of Food Engineering, London, v.40, n.1-2, p.9-13, 1999.

INCROPERA, F.P.; DE WITT, D.P. Fundamentals of heat and mass transfer. New York: John Willey, 1996. $886 \mathrm{p}$.

ITO, A.P.; AMENDOLA, M. Construção e avaliação de um sistema de determinação de condutividade e difusividade térmica em regime transiente. Simiente, Santiago, v.72, n.3-4, p.141-2, 2002.

MILES, C.A.; BEEK, G. van; VEERKAMP, C.H. Calculation of thermophisical properties, 1983. In: JANCSÓK, P.I.; SCHEERLINCK, N.; VERBOVEN, P.; NICOLAI, B.M.; DEBAERDEMAEKER, J. Shape modeling for stochastic FE computacion of cooling processes of agricultural products. $<$ http://ageng2002.szie.hu/programme> Acesso em: ago. 2002.

MITCHEL, F.G. Cooling horticultural commodities. In: KADER, A.A. Postharvest technology of horticultural crop. $2^{\text {nd }}$ ed. Davis: Cooperative Extension Division of Agricultural and Natural Resources, University of California, 1992. p.53-68.

PIROZZI, D.C.Z.; AMENDOLA, M. Resfriamento rápido com ar forçado de morangos: um estudo numérico usando método das diferenças finitas explícitas. In: CONGRESSO BRASILEIRO DE ENGENHARIA AGRÍCOLA, 31., 2002, Salvador. Anais.... Salvador: Sociedade Brasileira de Engenharia Agrícola, 2002. 1 CD ROM.

QUEIROZ, M.R.; NEBRA, S.A. Abordagem teórica e experimental do encolhimento na secagem de bananas. In: CONGRESSO BRASILEIRO DE ENGENHARIA AGRÍCOLA. 31., 2002, Salvador. Anais... Salvador: Sociedade Brasileira de Engenharia Agrícola, 2002. 1 CD ROM.

RICHTMYER, R.D.; MORTON, K.W. Difference methods for initial - value problems. New York: Interscience, 1967. $405 \mathrm{p}$.

TERUEL, M.B.J. Estudo teórico experimental do resfriamento de laranja e banana com ar forçado. 2000. 249 f. Tese (Doutorado em Engenharia Mecânica) - Faculdade de Engenharia Mecânica, Universidade Estadual de Campinas, Campinas, 2000.

TRELEA, I.C.; ALVAREZ, G.; TRYSTAM, G. Nonlinear predictive optimal control of a batch refrigeration process. Journal of Food Process Engineering, Westport, v.21, p.1-32, 1998. 